\title{
Influences des déplacements axiaux sur les caractéristiques du contact des engrenages spiro-coniques Klingelnberg
}

\author{
Márk Lelkes ${ }^{1}$, JÁnos Márialigeti ${ }^{2}$ et Daniel Play ${ }^{3}$ \\ 1 Département des Éléments et des Transmissions de Véhicules, Université des Sciences Techniques et Économique de Budapest, \\ Bertalan L. u. 2, 1111 Budapest, Hongrie \\ 2 Département des Éléments et des Transmissions de Véhicules, Université des Sciences Techniques et Économique de Budapest, \\ Bertalan L. u. 2, 1111 Budapest, Hongrie \\ 3 Institut National des Sciences Appliquées de Lyon, Bât. J. Verne, 19 Av. J. Capelle, 69621 Villeurbanne Cedex, France
}

Reçu le 4 décembre 2002, accepté le 10 juillet 2003

\begin{abstract}
Résumé - Une méthode numérique a été développée pour la simulation de la génération des dentures et du contact des engrenages spiro-coniques Klingelnberg. Les surfaces de denture sont corrigées suivant deux directions principales de la denture, soit en longueur, soit en hauteur. Les effets des désalignements axiaux sur les caractéristiques du contact issus de l'imprécision inévitable des pièces lors du montage dans les boîtes de transmission, sont déterminés.
\end{abstract}

Mots clés : Engrenage spiro-conique Klingelnberg / déterminations du contact entre dent / effet des modifications de profil et des mésalignements

\begin{abstract}
A method was developed for numerical simulation of generation and meshing of Klingelnberg bevel gears. Tooth surfaces were modified from theoretical shapes along both the width and height of teeth. Effects of axis misalignments due to assembly are also considered in order to defined tooth contact evolutions.
\end{abstract}

Key words: Klingelnberg bevel gear / tooth contact analysis / tooth profile modification / misalignment effect

\section{Introduction}

La conception préliminaire des transmissions de puissance par engrenages demande une définition précise de la géométrie afin d'assurer un bon fonctionnement cinématique et dynamique. Le comportement des transmissions avec engrenages spiro-coniques est particulièrement sensible à la définition initiale de la surface de denture. Déjà, plusieurs auteurs ont donné une définition formalisée de la géométrie [1-5], en tenant compte des paramètres de taillage significatifs $[2,5-7]$. Dans la pratique, l'aire de contact doit être située au milieu de la surface du flanc de denture pour présenter des conditions de contact satisfaisantes et un niveau d'erreur cinématique le plus petit possible. Ainsi des corrections de surfaces sont réalisées soit le long de la denture, soit en hauteur (piedtête) [3,4]. L'influence des modifications géométriques sur les paramètres de taillage de base sur le changement de la géométrie de la surface n'est pas facile à estimer. Les deux surfaces de denture, celle du pignon et celle de la roue, sont modifiées. En conséquence, les paramètres de taillage du pignon et de la roue, de même que les désalignements axiaux doivent être considérés pour étudier l'engrènement. Dans les applications, sous charge, les surfaces réelles s'écartent des surfaces théoriques de denture conjuguées, pour éviter le contact aux parties extrêmes de la denture (pied et tête), et assurer ainsi une portée localisée dans un voisinage du centre des flancs. Cet écart des surfaces réelles est assuré par des corrections de dentures, en utilisant des réglages de machine appropriés. Mais il faut éviter d'obtenir une erreur cinématique non-continue en fonction du temps [3, 5, 8-10], tout en assurant une portée localisée. La cause de l'erreur cinématique discontinue peut être double. L'ensemble des corrections et des modifications peut conduire à une interruption de l'engrènement [10]. Une autre cause de fonction discontinue d'erreur cinématique est le désalignement des engrenages lors du montage dans les boîtes de transmission [3]. Ainsi les surfaces de denture sont corrigées suivant deux directions pour obtenir à la fois, 
une erreur cinématique continue et une portée localisée. L'étude présentée ici concerne les engrenages spiro-coniques Klingelnberg qui sont appliquée majoritairement aux boîtes de transmission des véhicules (automobile ou rail). Elle a pour but de qualifier les effets des désalignements d'axes sur les caractéristiques du contact, afin de prévoir les réglages de la machine de taillage et de réduire le temps de mise au point du couple d'engrenage pour un bon fonctionnement.

\section{Simulation du contact [11]}

La simulation de contact est basée sur la théorie de la tangence continuelle des surfaces de denture en contact. Elle est obtenue à partir d'une simulation numérique de la génération simultanée des surfaces en contact : la surface convexe du pignon et la surface concave de la roue. Les deux surfaces satisfaisant les équations de génération, sont présentées dans le repère $S_{s}$ (Fig. 1). Ces surfaces idéales ont un point commun dans la position de contact (Eq. (1)) et les vecteurs unitaires normaux des surfaces sont colinéaires (Eq. (2))

$$
\begin{array}{r}
\mathbf{r}_{s 1}\left(t_{1}, \varphi_{1}, \psi_{1}, \phi_{1}\right)-\mathbf{r}_{s 2}\left(t_{2}, \varphi_{2}, \psi_{2}, \phi_{2}\right)=0 \\
\mathbf{n}_{s 1}\left(t_{1}, \varphi_{1}, \psi_{1}, \phi_{1}\right)-\mathbf{n}_{s 2}\left(t_{2}, \varphi_{2}, \psi_{2}, \phi_{2}\right)=0
\end{array}
$$

\subsection{Détermination de la portée}

Dans le cas du contact sous charge, des déformations se produisent et les aires de contact assimilées à des ellipses peuvent être calculées par la théorie de Hertz. Durant l'engrènement, les aires de contact se déplacent et l'ensemble de leur position définit une portée. Pour simplifier l'approche et pour obtenir des indications rapides sur les réglages de fabrication, seule la cinématique des corps solides est considérée. La simulation du contact considère une approximation géométrique des ellipses de contact qui correspond à un décalage théorique de surface de denture. Dans la pratique, cette méthode repose sur la détermination de la distance entre les surfaces en contact. Il faut noter que cette distance est petite. Les points ainsi obtenus forment le bord de l'ellipse. À l'intérieur de cette ellipse, la distance entre les deux surfaces en contact, est inférieure à une distance choisie.

Une méthode est proposée [11] pour réaliser une approximation du calcul de la distance entre les surfaces de denture dans la détermination des ellipses de contact. Pour une position instantanée de contact d'engrènement, les surfaces de denture sont fixées dans le repère $S_{s}$ (Fig. 1) à une distance relative fixée à $10 \mu \mathrm{m}$. Dans la proximité du point de contact $P_{s j}$, les plans successifs et perpendiculaires à l'axe $z_{s}$ sont considérés (Fig. 2a). Dans chaque plan, les couples de points $\left(P_{s 1}, P_{s 2}\right)$ sont recherchés tels que la distance $\left|\overline{P_{s 1 j} P_{s 2 j}}\right|$ soit égale à $10 \mu \mathrm{m}$ et tels que les longueurs $\left|\mathbf{r}_{s j 1}\right|=\left|\mathbf{r}_{s j 2}\right|$ soient égales. L'ellipse de contact est présentée figure $2 \mathrm{~b}$. Cette ellipse se déplace suivant la ligne de contact. L'orientation de l'ellipse par rapport à la ligne de contact varie très peu au cours de l'engrènement.

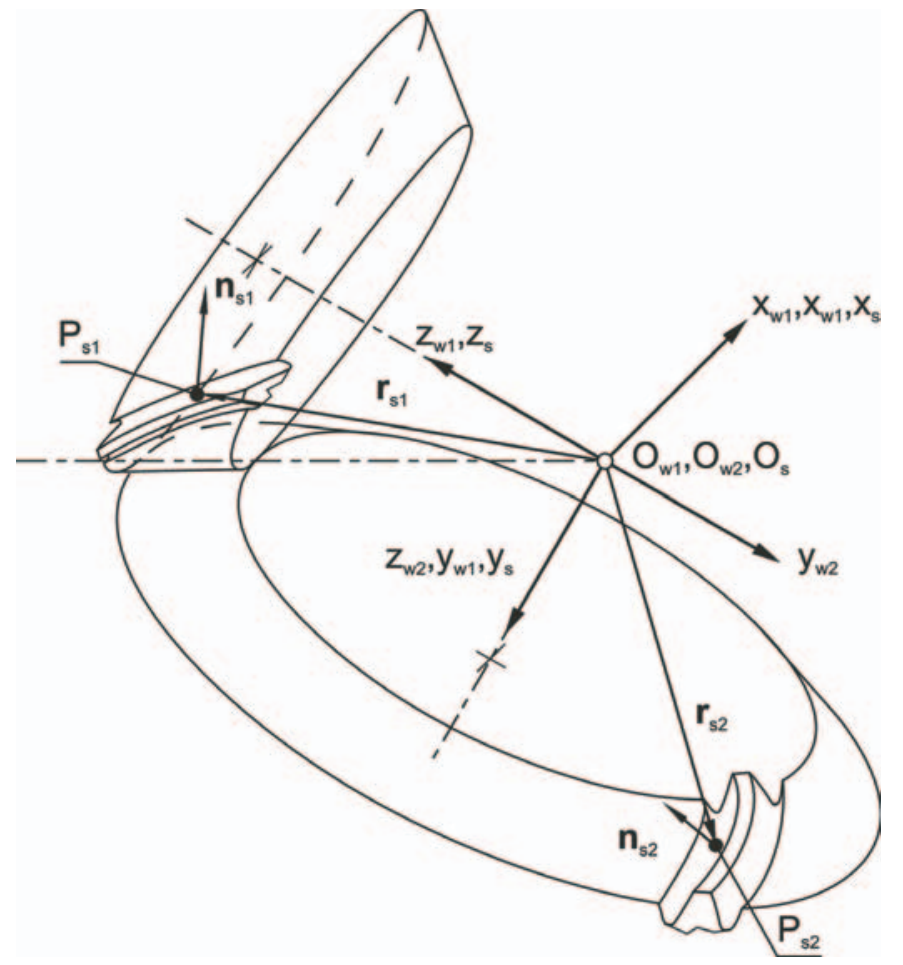

Fig. 1. Repères pour la définition du contact.

\subsection{Visualisation de la portée}

La portée est présentée sur la surface du flanc de denture du pignon (Fig. 3). La ligne de contact est composée des points de contact instantanés (en ligne grasse sur la figure). La présentation de l'ellipse de contact est réduite à un segment qui correspond au grand axe de l'ellipse. La partie active de la portée est déterminée par l'entrée et la sortie de l'engrènement.

Trois points de référence sont définis sur la surface de denture du pignon. Les caractéristiques du contact sont déterminées à partir de ces points :

- $M$, intersection de la ligne de contact et du cône primitif ou complémentaire,

- $C$, point central de la surface de denture, c'est l'intersection des cônes primitif et complémentaire,

- $T$, référence au sommet de la courbe parabolique de la fonction de l'erreur cinématique sur la ligne de contact. Ce point est repéré par ses coordonnées dans un repère $(C, x, y)$ d'origine le point $C$.

Le déplacement de la portée sur le cône primitif est donné par la distance $(M, C)$. La position du point $T$ dans le repère $(C, x, y)$ détermine le déplacement de la partie active de la portée. L'erreur cinématique nulle est fixée au point de courbe maximale de sa fonction et qui correspond à l'instant de l'engrènement du point $M$. La largeur de la portée est déterminée par la longueur du grand axe de l'ellipse au point $M$. 


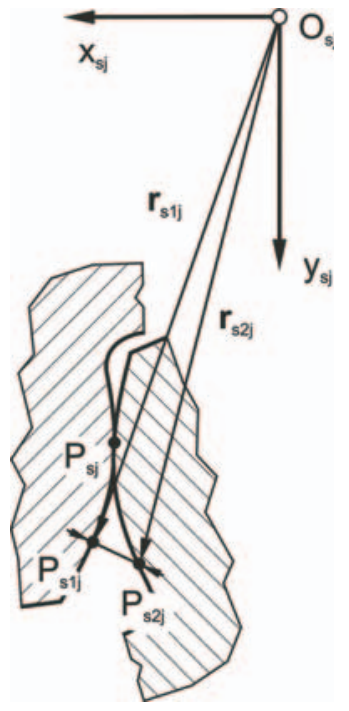

(a)

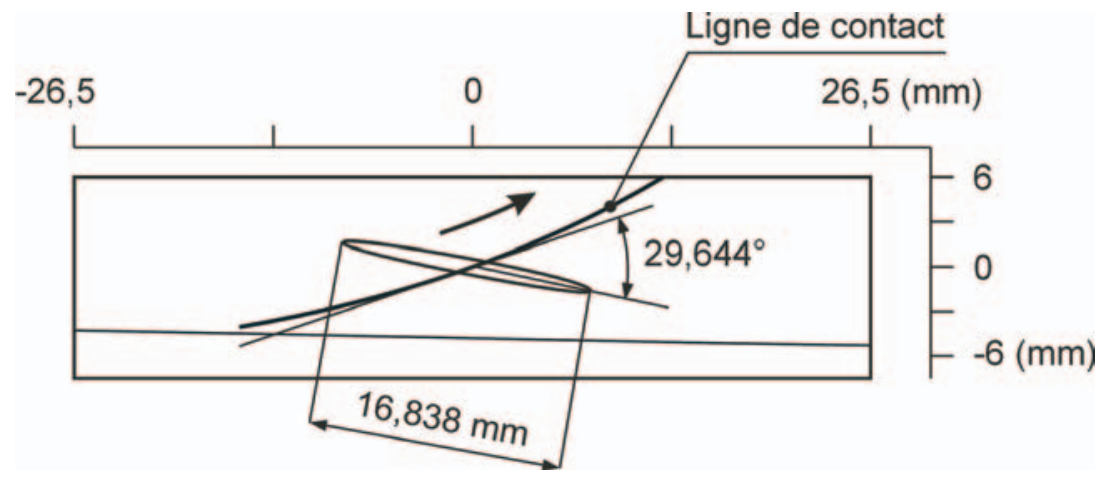

(b)

Fig. 2. Définition de l'ellipse de contact. (a) Détermination de l'ellipse de contact dans un plan perpendiculaire à l'axe $z_{s j}$. (b) Dimensions de l'ellipse de contact pour un point de fonctionnement.

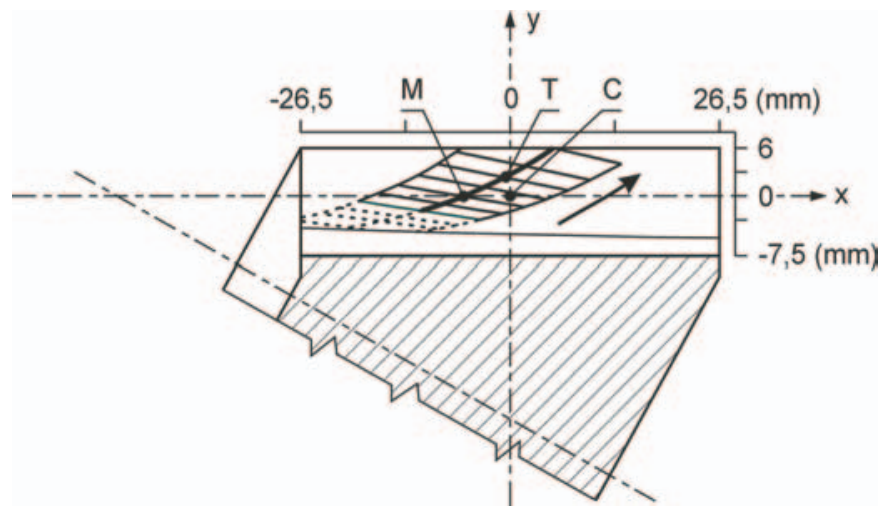

Fig. 3. Localisation de la portée sur la surface de denture du pignon.

\section{Méthode d'investigation}

Les données initiales issues de la géométrie des engrenages spiro-coniques du système Klingelnberg ont été considérées (Tab. 1). Les paramètres de taillage des
Tableau 1. Données initiales.

\begin{tabular}{llcc} 
& & Pignon & Roue \\
\cline { 3 - 4 } Nombre de dents & $N$ & 19 & 34 \\
Angle des axes & $\left(^{\circ}\right)$ & 90 & \\
Angle primitif $\delta_{m}$ & $\left(^{\circ}\right)$ & 29,197 & 60,803 \\
Angle de spirale $\beta_{m}$ & $\left(^{\circ}\right)$ & 29,686 & \\
Direction de spirale & & Gauche & Droite \\
Génératrice moyenne $R_{m}$ & $(\mathrm{~mm})$ & 136,74 & 136,74 \\
Génératrice primitive $R_{e}$ & $(\mathrm{~mm})$ & 163,24 & 163,24 \\
Largeur de denture $b$ & $(\mathrm{~mm})$ & 53 & 53 \\
Hauteur $(\mathrm{mm})$ & & 13,5 & 13,5 \\
Module normale $m_{n}$ & $(\mathrm{~mm})$ & 6,1 & 6,1 \\
\hline
\end{tabular}

surfaces des dentures de base qui sont appliqués dans les simulations, sont définis pour le pignon (Tab. 2) et pour la roue (Tab. 3). Seules les surfaces principales, le flanc convexe du pignon et le flanc concave de la roue, sont concernées. 
Tableau 2. Paramètres de taillage liés au pignon.

\begin{tabular}{|c|c|c|c|c|c|}
\hline & & \multicolumn{4}{|c|}{ Types de correction } \\
\hline & & Aucune & $\begin{array}{c}\text { En } \\
\text { longueur } \\
(\mathrm{A} 1)\end{array}$ & $\begin{array}{c}\text { En } \\
\text { hauteur } \\
(\mathrm{B} 1)\end{array}$ & $\begin{array}{c}\text { Deux } \\
\text { directions } \\
(\mathrm{C} 1)\end{array}$ \\
\hline Rayon de l'outil coupant $R_{b}$ & $(\mathrm{~mm})$ & $\infty$ & $\infty$ & 509,634 & 509,634 \\
\hline Angle de base d'outil $\nu$ & $\left({ }^{\circ}\right)$ & 6,372 & 6,486 & 6,372 & 6,486 \\
\hline Rayon de la fraise $R_{h}$ & $(\mathrm{~mm})$ & 137,4 & 135 & 137,4 & 135 \\
\hline Distance machine $M_{d}$ & $(\mathrm{~mm})$ & 150,83 & 149,595 & 150,683 & 149,595 \\
\hline Rayon du cercle roulant $\rho$ & $(\mathrm{mm})$ & 133,54 & 132,576 & 133,54 & 132,576 \\
\hline Rayon du cercle de base $p$ & $(\mathrm{~mm})$ & 17,143 & 17,019 & 17,143 & 17,019 \\
\hline Module de l'outil coupant $m_{0}$ & $(\mathrm{~mm})$ & 6 & 6 & 6 & 6 \\
\hline Angle de pression $\alpha_{n}$ & $\left({ }^{\circ}\right)$ & 20 & 20 & 20 & 20 \\
\hline Rayon de la tête de l'outil coupant $R_{c}$ & $(\mathrm{~mm})$ & 1,8 & 1,8 & 1,8 & 1,8 \\
\hline Nombre de groupes coupant & & 5 & 5 & 5 & 5 \\
\hline
\end{tabular}

Tableau 3. Paramètres de taillage liés à la roue.

\begin{tabular}{llc}
\hline Rayon de l'outil coupant $R_{b}$ & $(\mathrm{~mm})$ & $\infty$ \\
Angle de base d'outil $\nu$ & $\left({ }^{\circ}\right)$ & 6,372 \\
Rayon de la fraise $R_{h}$ & $(\mathrm{~mm})$ & 137,4 \\
Distance machine $M_{d}$ & $(\mathrm{~mm})$ & 150,83 \\
Rayon du cercle roulant $\rho$ & $(\mathrm{mm})$ & 133,54 \\
Rayon du cercle de base $p$ & $(\mathrm{~mm})$ & 17,143 \\
Module de l'outil coupant $m_{0}$ & $(\mathrm{~mm})$ & 6 \\
Rayon de la tête de l'outil coupant $R_{c}$ & $(\mathrm{~mm})$ & 1,8 \\
Nombre de groupes coupant & & 5 \\
\hline
\end{tabular}

Pour les surfaces convexes du pignon, des surfaces de denture sans correction, avec une correction en longueur, avec une correction en hauteur et avec une double correction (en longueur et en hauteur) sont successivement considérées. Ainsi, plusieurs combinaisons de surfaces de denture ont été étudiées. Trois cas de base ont été considérés selon le type de correction :

- A1, correction suivant la longueur de la denture,

- B1, correction suivant la hauteur de la denture,

- C1, double correction.

\section{Contact des dentures corrigées}

Plusieurs réglages de machine peuvent être considérés pour réaliser des corrections. Les corrections sont faites soit suivant la longueur, soit suivant la hauteur de la dent. Les surfaces non-conjuguées sont utilisées pour éviter le contact aux extrémités de la denture, notamment les contacts de crête au petit bout, au gros bout et à la tête. Le contact des surfaces conjuguées, initialement linéique, devient alors un contact ponctuel avec l'enlèvement de matière dû aux corrections. Dans le même temps, les corrections entraînent une erreur cinématique dont la variation au cours du temps est une fonction parabolique.
La correction en longueur est réalisée par une variation du rayon $R_{h}$ de la fraise outil de taillage (Fig. 4a). Un outil avec une arête de coupe courbe $\left(R_{b}\right)$ remplace l'outil coupant d'arête rectiligne, afin de modifier en hauteur la surface de denture (Fig. 4b).

Avec la correction en longueur, l'aire de contact évolue suivant le profil à la surface de denture. Pour la correction en hauteur, un contact longitudinal apparaît. Pour une double correction, une portée diagonale et une forme parabolique de la fonction de l'erreur cinématique sont obtenues. Tous ces effets sont étudiés en détail dans les sous paragraphes suivants.

\subsection{Effets des corrections en longueur $[10,11]$}

Dans le cas A1, l'angle de rotation $\kappa$ de l'outil coupant est égal à zéro. Initialement, le plan de l'outil coupant est dirigé au centre de rotation instantané dans la position centrale de la génération au plan primitif (Fig. 4a). Il n’y a pas d'erreur cinématique $\Delta \phi_{2}$ (écart de position de la roue menée par rapport à la valeur théorique en fonction de la position angulaire $\phi_{1}$ du pignon menant) parce que les points conjugués restent le long d'une ligne unique. Un premier type de contact traversant la surface de denture suivant le profil se produit (Fig. 5a).

Le contact sur les crêtes est étudié (Fig. 5a). Le contact est divisé en trois segments $a, b$ et $c$. Les segments $a$ et $c$ sont liés aux contacts de crête. Le segment $a$ est situé à la partie basse de la pièce menante, et sur la crête de tête de la pièce menée. L'erreur cinématique lorsque le point de contact n'est pas sur la partie active de la denture (segment $b$ ) augmente excessivement (segments $a$ et $c$ ) (Fig. 5b). Ce phénomène n'apparaît que si un seul couple de dents est en contact. Dans la pratique, plusieurs couples de dents sont actifs, ainsi le contact de crête ne peut pas être établi. Le premier couple de dents a toujours une erreur cinématique nulle quand le deuxième couple de dent est déjà au contact avec une erreur cinématique nulle. Le contact se réalise toujours 


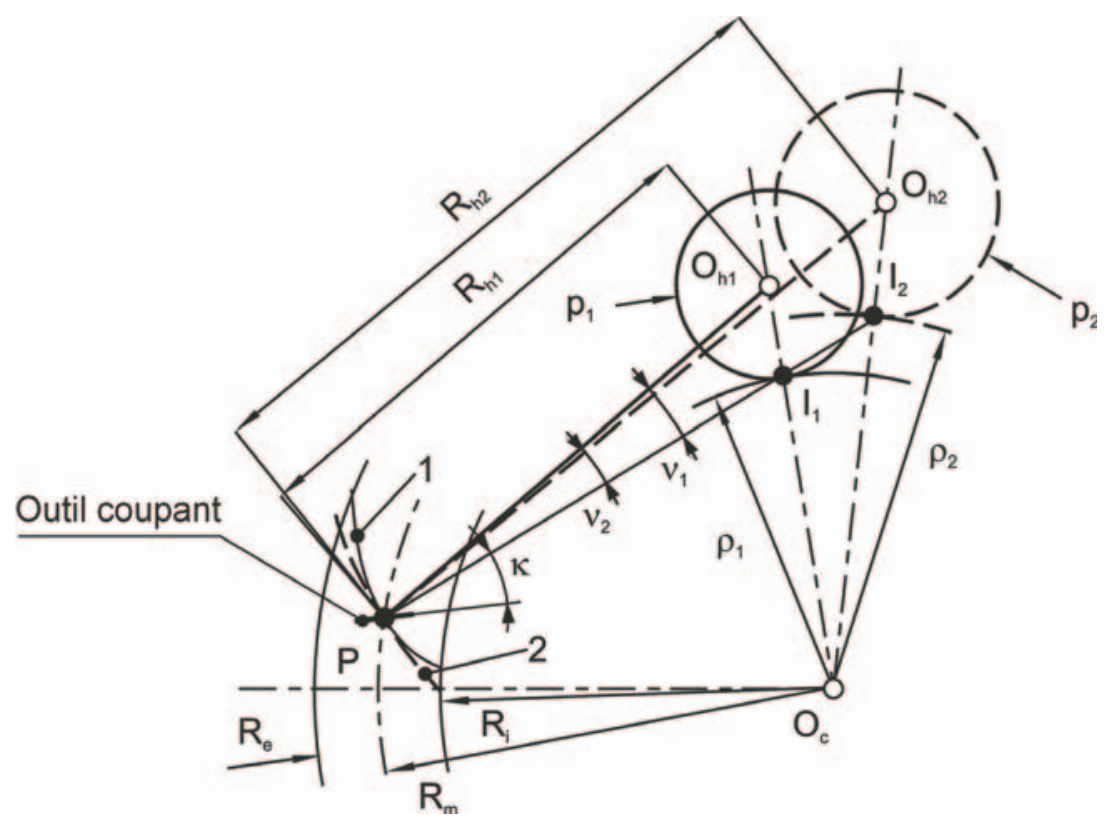

(a)

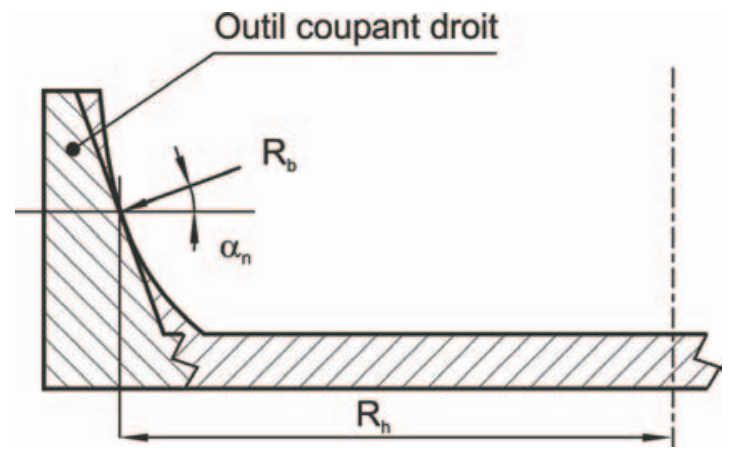

(b)

Fig. 4. Définition des paramètres pour les corrections de denture. (a) Corrections en longueur. (b) Correction en hauteur.

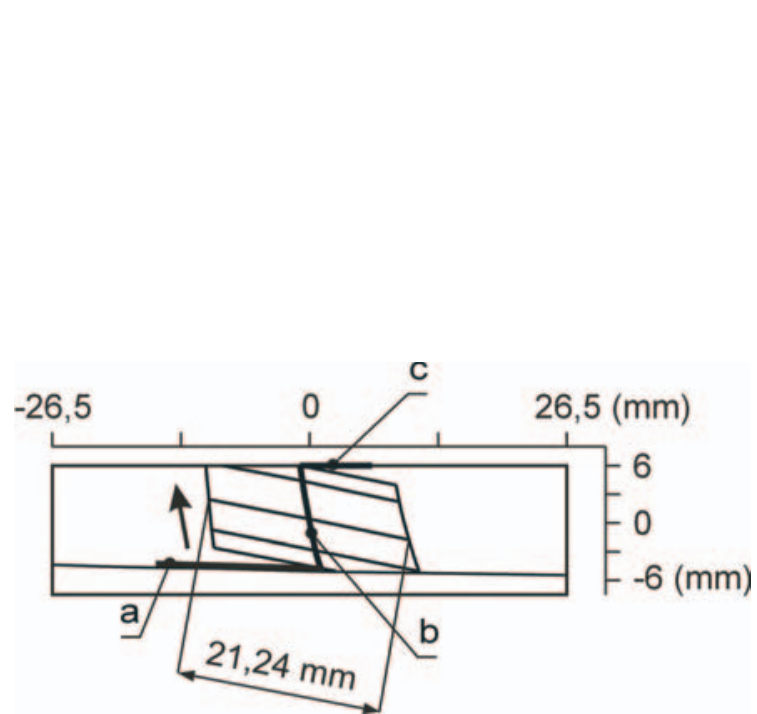

(a)

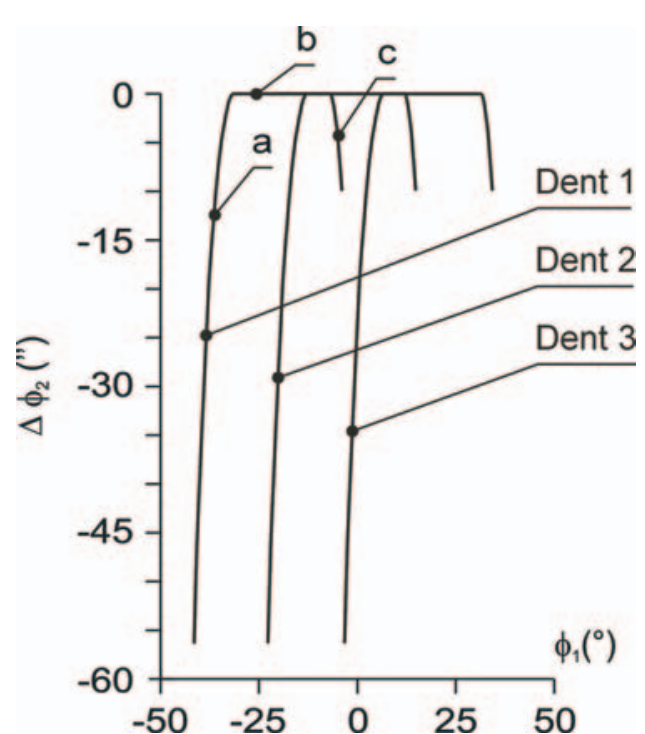

(b)

Fig. 5. Visualisation des résultats pour le cas A1. (a) Portée. (b) Erreur cinématique. 


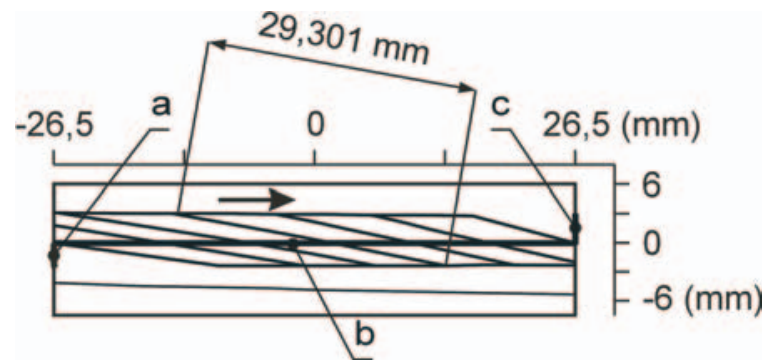

(a)

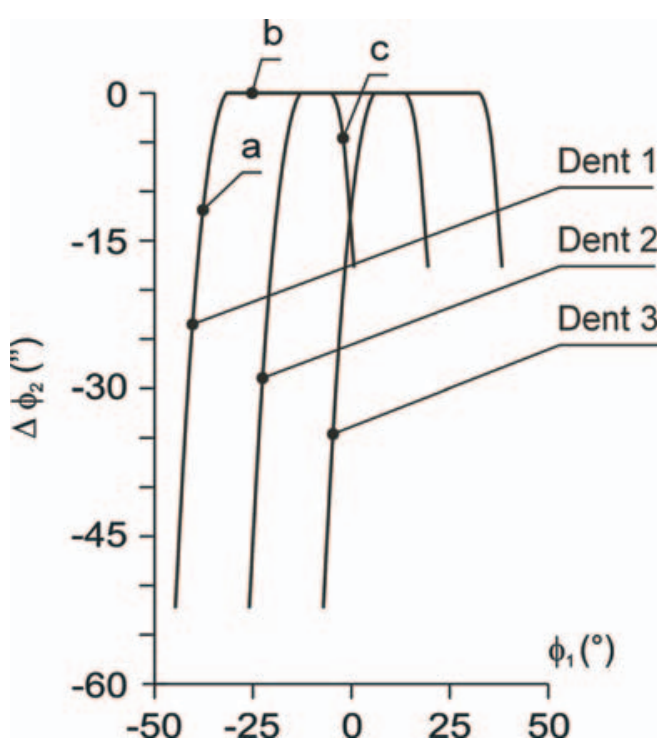

(b)

Fig. 6. Visualisation des résultats pour le cas B1. (a) Portée. (b) Erreur cinématique.

entre les couples de dents qui ont le niveau de l'erreur cinématique le plus bas. Pour le cas A1 exposé ice, la largeur de la portée est égale à 21,24 millimètres alors qu'avec les cas B1 et $\mathrm{C} 1$ les largeurs sont respectivement de 29,30 et $16,84 \mathrm{~mm}$.

\subsection{Effets des corrections en hauteur $[10,11]$}

Dans le cas B1, l'angle de rotation $\kappa$ de l'outil coupant est égal à zéro. Il n'y a pas d'erreur cinématique si la surface active de la denture est en contact. Les points conjugués restent le long d'une ligne unique. Cette particularité est commune avec la correction en longueur vue précédemment. La ligne de contact a une orientation (Fig. 6a) inchangée sous l'effet des déplacements d'axe.

Le contact sur les crêtes aux extrémités de la surface de denture est étudié (Fig. 6a). Le contact est divisé en trois segments $a, b$ et $c$. Les segments $a$ et $c$ sont liés aux contacts en bout. La partie $a$ est située au petit bout de la pièce menante et sur la crête au petit bout de la pièce menée. L'erreur cinématique à l'exception de la partie active de la denture (segment $b$ ) augmente excessivement (Fig. 6b). Cette variation apparaît lorsqu'un seul couple de dents est considéré. Mais dans le cas de plusieurs dents, le contact se réalise pour un niveau d'erreur cinématique le plus bas.

\subsection{Effets des corrections suivant deux directions $[10,11]$}

Lorsque le flanc de la denture est corrigé suivant deux directions (cas C1), il ne reste qu'un seul point conjugué. En comparant un flanc de denture ayant subi des corrections par rapport au flanc de denture initial, les points qui ont la même définition géométrique (position et normale) dans la position de génération sont nommés conjugués. La fonction erreur cinématique a une forme quasi parabolique (Fig. 7b). La nouvelle ligne de contact est influencée par les deux corrections. Elle est située entre les lignes de contact des deux cas précédents (cas A1 et cas B1) (Fig. 7a). La ligne de contact s'approche du cône primitif lorsque le rayon de la fraise $R_{h}$ augmente. Une portée diagonale est obtenue avec les réglages de machine examinés. La valeur maximum de l'erreur cinématique $\Delta \phi_{2 \max }$ calculée pour le cas C1 est égale à $-23,604^{\prime}$.

\subsection{Conclusion sur les effets des corrections}

L'objectif du travail était de simuler numériquement l'influence des paramètres de fabrication sur la définition des portées et sur l'erreur cinématique des engrenages Klingelnberg. On constate que :

- de faibles modifications des paramètres de taillage modifient beaucoup la portée et l'erreur cinématique. On doit donc contrôler dans le détail ces paramètres de taillage et suivre leur évolution au cours du temps;

- une optimisation organisée des portées est réalisable à partir des simulations numériques. On peut donc éviter une recherche expérimentale des portées avec une roue et un pignon réels.

\section{Influence des déplacements d'axes sur les caractéristiques du contact}

L'imprécision inévitable de l'alignement des engrenages lors du montage dans les boîtes de transmission nécessite des études sur les effets des variations d'alignements des axes. Ces variations d'alignement sont dues à 


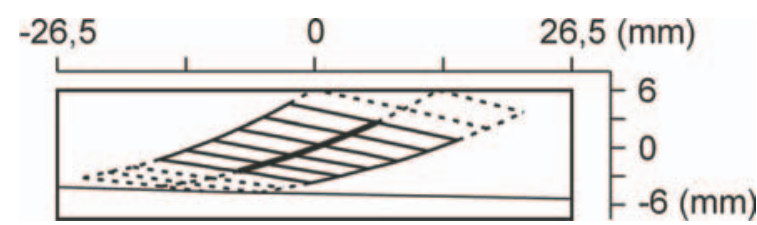

(a)

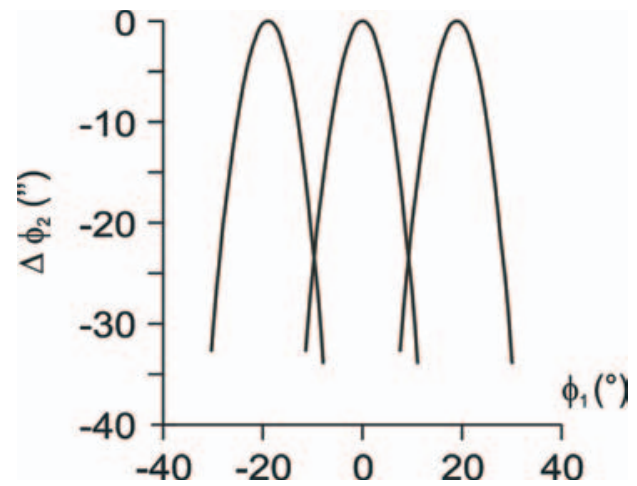

(b)

Fig. 7. Visualisation des résultats pour le cas C1. (a) Portée. (b) Erreur cinématique.

Tableau 4. Définition des cas examinés, désalignements d'axes.

\begin{tabular}{ccccc} 
& & \multicolumn{3}{c}{ Types de correction } \\
\cline { 2 - 5 } Type de & Valeur de & En & En & Deux \\
désalignement & déplacement & longueur & hauteur & directions \\
\hline Décalage & $+0,1 \mathrm{~mm}$ & $\mathrm{~A} 2$ & $\mathrm{~B} 2$ & C2 \\
hypoïde & $-0,1 \mathrm{~mm}$ & A3 & B3 & C3 \\
\hline Déplacement & $+0,1 \mathrm{~mm}$ & A4 & B4 & C4 \\
axial de la roue & $-0,1 \mathrm{~mm}$ & A5 & B5 & C5 \\
\hline Déplacement & $+0,1 \mathrm{~mm}$ & A6 & B6 & C6 \\
axial du pignon & $-0,1 \mathrm{~mm}$ & A7 & B7 & C7 \\
\hline Variation de & $+10^{\prime}$ & A8 & B8 & C8 \\
l'angle primitif & $-10^{\prime}$ & A9 & B9 & C9
\end{tabular}

la fabrication et au montage de même qu'aux effets des déformations dues aux charges appliquées. La méthode de simulation et visualisation des portées va donc être appliquée pour étudier l'influence des déplacements d'axes. En particulier, l'objectif est de prévoir les nouveaux paramètres de machines à appliquer pour réaliser des corrections de denture qui donneront une bonne portée et une erreur cinématique minimale.

\subsection{Méthode d'investigation et résultats}

Pour la surface de denture du pignon, les corrections en longueur, en hauteur et suivant les deux directions sont considérées. Dans la réalité, un grand nombre de positionnements de pièces peut être défini, mais les déplacements suivants ont été pris en compte (Fig. 8) :

- décalage hypoïde (offset);

- déplacement axial de la roue;

- déplacement axial du pignon;

- variation de l'angle primitif.

Ces déplacements sont considérés dans les 2 sens. Huit cas sont ainsi ajoutés aux corrections de référence (en longueur, en hauteur et suivant deux directions) déjà traitées. Les cas étudiés sont présentés dans le tableau 4.
Tableau 5. Changement de position et de la largeur de la portée, valeur du saut cinématique, cas A.

\begin{tabular}{cccc}
\hline Cas & $\begin{array}{c}\text { Changement } \\
\text { de position } \\
\text { de la portée } \\
s_{w}(\mathrm{~mm})\end{array}$ & $\begin{array}{c}\text { Largeur de } \\
\text { la portée } \\
w \\
(\mathrm{~mm})\end{array}$ & $\begin{array}{c}\text { Saut de } \\
\text { l'erreur } \\
\text { cinématique } \\
j\left({ }^{\prime}\right)\end{array}$ \\
\hline A2 & 4,783 & 21,223 & 16,755 \\
A3 & $-4,962$ & 21,272 & 14,642 \\
A4 & 0,936 & 21,337 & 13,307 \\
A5 & $-0,892$ & 21,160 & 13,856 \\
A6 & 3,487 & 21,041 & 23,407 \\
A7 & $-3,511$ & 21,455 & 25,304 \\
A8 & 3,478 & 24,744 & 23,877 \\
A9 & $-3,606$ & 23,594 & 29,919 \\
\hline
\end{tabular}

Finalement, vingt-quatre combinaisons (cas A2-9, B2-9, C2-9) ont été examinées et comparées.

Le sens de déplacement de la portée dépend de la direction du désalignement. Des valeurs de $-0,1 \mathrm{~mm}$ et $+0,1 \mathrm{~mm}$ ou $-10^{\prime}$ et $+10^{\prime}$ sont considérées, des différences considérables se produisent dans le changement de position de la portée (Tabs. 5-7 liés respectivement aux cas A, $\mathrm{B}$ et $\mathrm{C}$ ). Les déplacements des axes font déplacer la portée sur la surface du pignon vers le petit bout et vers la tête, si le désalignement est négatif. Si le désalignement est 


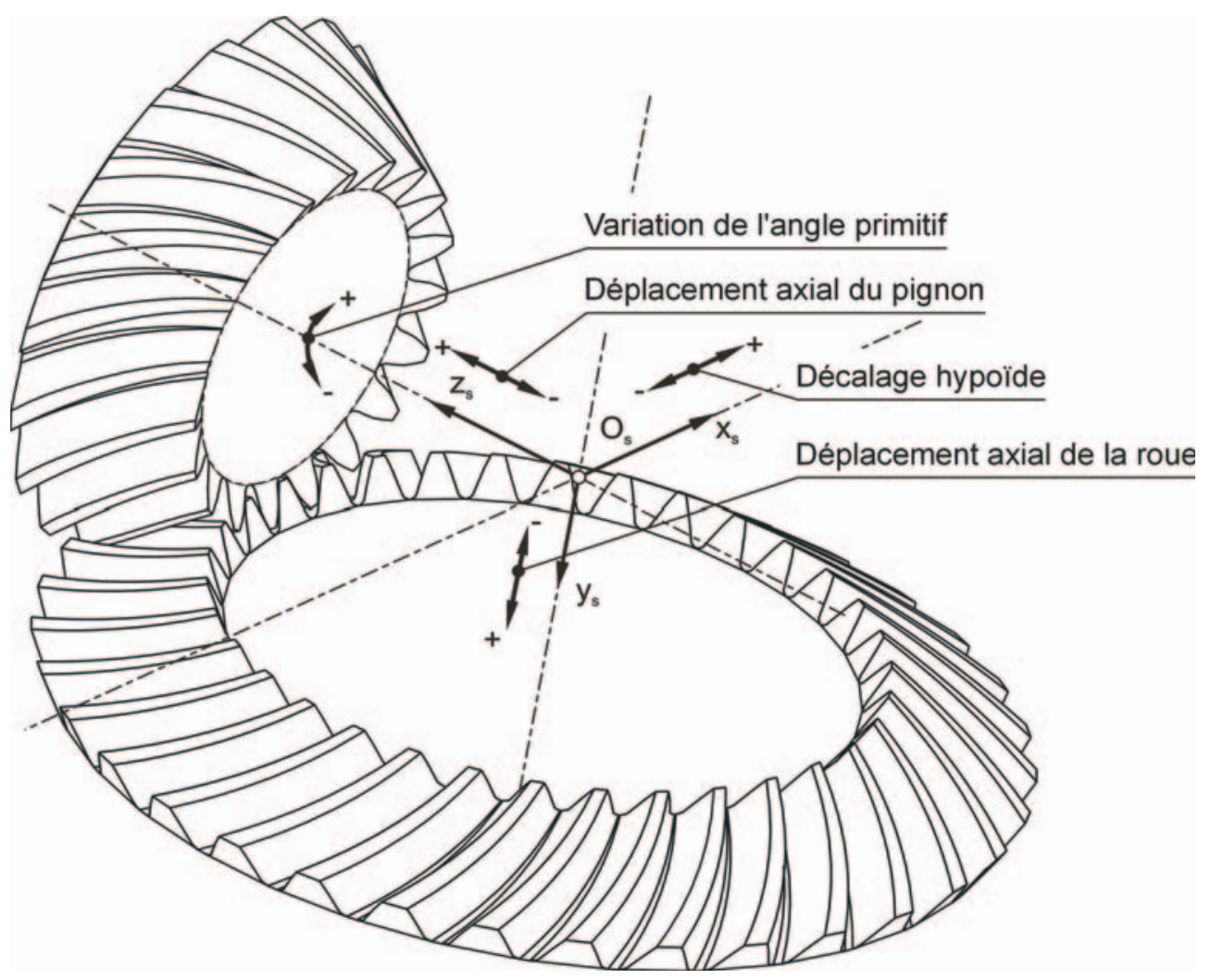

Fig. 8. Définition des déplacements axiaux.

Tableau 6. Changement de position et de la largeur de la portée, valeur du saut cinématique, cas B.

\begin{tabular}{cccc}
\hline Cas & $\begin{array}{c}\text { Changement } \\
\text { de position } \\
\text { de la portée } \\
s_{h}(\mathrm{~mm})\end{array}$ & $\begin{array}{c}\text { Largeur de } \\
\text { la portée } \\
w \\
(\mathrm{~mm})\end{array}$ & $\begin{array}{c}\text { Saut de } \\
\text { l'erreur } \\
\text { cinématique } \\
j\left(^{\prime}\right)\end{array}$ \\
\hline B2 & $-1,490$ & 24,151 & 38,733 \\
B3 & 1,935 & - & 49,384 \\
B4 & $-0,304$ & 28,386 & 3,240 \\
B5 & 0,330 & 30,386 & - \\
B6 & $-1,111$ & 25,076 & 17,955 \\
B7 & 1,422 & - & 25,616 \\
B8 & $-1,501$ & - & 11,428 \\
B9 & 1,860 & - & - \\
\hline
\end{tabular}

positif, le déplacement de la portée se fait dans le sens opposé (gros bout ou pied). Dans les trois cas (A, B, C), le déplacement axial de la roue a le moins d'influence sur la position de la portée. Les valeurs absolues des déplacements de la portée sont quasi-égales en fonction du type de déplacement, mais leurs directions sont opposées. Pour le cas C, le déplacement de la portée est bien décrit par les valeurs des cordonnées du point $T$ correspondant au sommet de la courbe de l'erreur cinématique. À partir de la position de ce point, le déplacement actif de la portée est donné.

La variation de la largeur de la portée est de 16,7\% dans les cas A (Tab. 5), 25,8 \% dans les cas B (Tab. 6) et $23 \%$ dans les cas C (Tab. 7). Dans certains cas (B3,
B7-9), l'extrémité de la portée est en dehors de la surface active ainsi la largeur de la portée n'a pas pu être définie.

\subsection{Synthèse des effets des déplacements avec correction de denture}

Les désalignements à valeurs négatives déplacent la portée du pignon (cas A3, A5, A7 et A9) vers le petit bout de la denture (Fig. 10a), et vers le gros bout pour des valeurs positives (Fig. 9a) (cas A2, A4, A6 et A8) (Tab. 5). Les figures correspondant aux cas A2-A7 sont présentées dans l'Annexe B. Les désalignements entraînent une discontinuité de l'erreur cinématique, ainsi un saut de l'erreur cinématique apparaît. L'engrènement se réalise sur le couple de dents instantané qui a l'erreur cinématique la plus grande. En conséquence, si la roue menée est en avance par rapport au pignon, le saut cinématique passe d'un niveau plus petit à un niveau plus grand dès que le couple de dents suivant rentre au contact (Fig. 10b). Ce phénomène a le plus mauvais effet sur la cinématique. Si la roue menée est en retard (Fig. 9b), le saut va d'un niveau plus grand à un niveau plus petit. Le sens de déplacement et la pente de la fonction erreur cinématique dépendent de la direction du déplacement. La roue menée est en avance par rapport à la vitesse angulaire idéale si le déplacement est positif (cas A2, A4, A6). La rotation peut être négative dans le cas A9 (Fig. 9b). Dans les cas A3, A5, A7 et A8, la roue menée est en retard par rapport au pignon menant. La plus grande variation de l'erreur cinématique est 


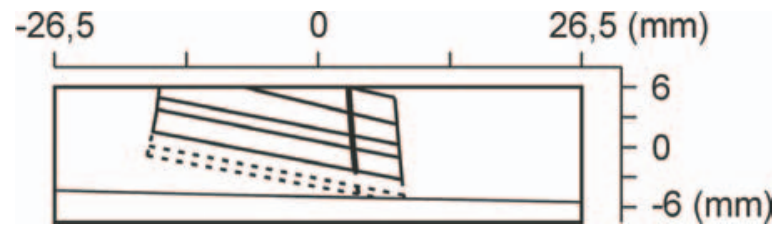

(a)

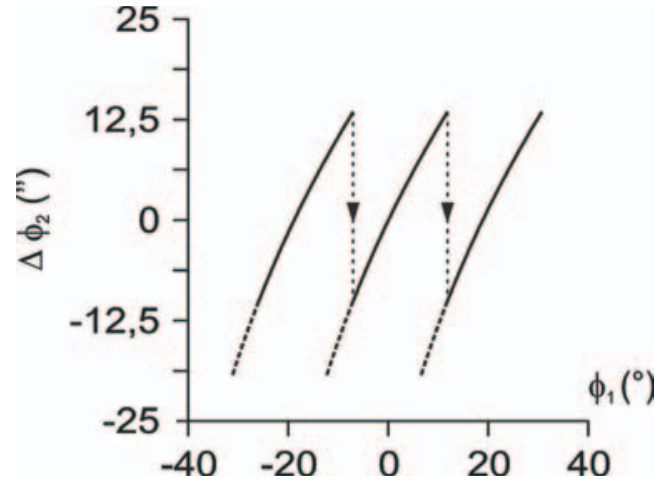

(b)

Fig. 9. Visualisation des résultats pour le cas A8. (a) Portée. (b) Erreur cinématique.

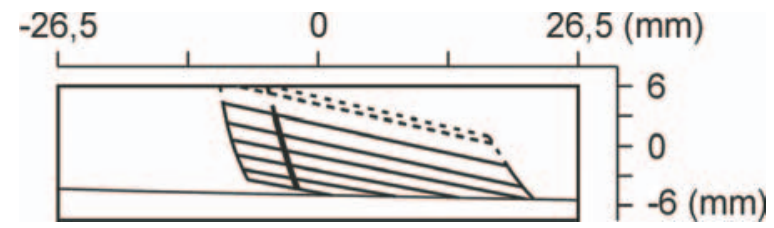

(a)

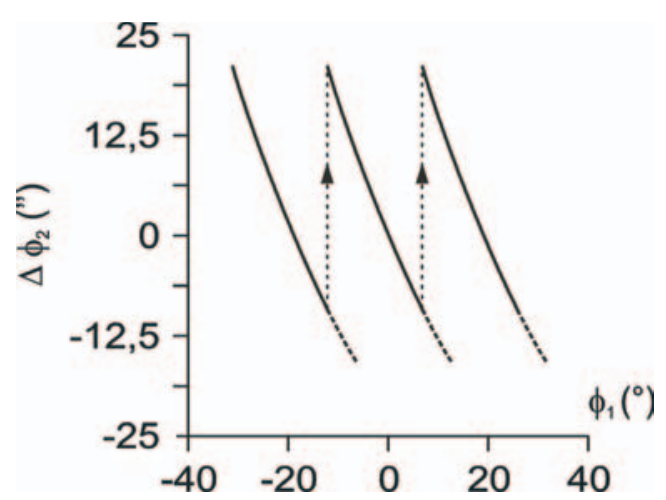

(b)

Fig. 10. Visualisation des résultats pour le cas A9. (a) Portée. (b) Erreur cinématique.

Tableau 7. Changement de position de la portée, largeur de la portée, coordonnées du point $T$, erreur cinématique maximale, cas C.

\begin{tabular}{|c|c|c|c|c|}
\hline Cas & $\begin{array}{c}\text { Changement } \\
\text { de position } \\
\text { de la portée } \\
s_{w}(\mathrm{~mm})\end{array}$ & $\begin{array}{c}\text { Largeur } \\
\text { de la portée } \\
w \\
(\mathrm{~mm})\end{array}$ & $\begin{array}{c}\text { Point } T(x, y) \\
\text { cordonnée } \\
(\mathrm{mm}) \\
\end{array}$ & $\begin{array}{c}\text { Erreur } \\
\text { cinématique } \\
\text { maximale } \\
\Delta \phi_{2 \max }\left(^{\prime}\right)\end{array}$ \\
\hline $\mathrm{C} 2$ & 4,783 & 16,353 & 3,$618 ;-0,473$ & $-23,304$ \\
\hline $\mathrm{C} 3$ & $-4,962$ & 17,303 & $-3,892 ; 0,420$ & $-22,543$ \\
\hline $\mathrm{C} 4$ & 0,735 & 16,770 & $-0,021 ;-0,260$ & $-22,787$ \\
\hline $\mathrm{C} 5$ & $-0,718$ & 16,882 & $-0,029 ; 0,275$ & $-23,834$ \\
\hline $\mathrm{C} 6$ & 3,7169 & 16,409 & 1,$783 ;-0 ; 644$ & $-21,382$ \\
\hline $\mathrm{C} 7$ & $-3,719$ & 17,198 & $-1,985 ; 0,729$ & $-22,105$ \\
\hline $\mathrm{C} 8$ & 4,661 & 20,115 & 2,$434 ;-0,777$ & $-21,463$ \\
\hline $\mathrm{C} 9$ & $-4,709$ & 19,711 & $-2,865 ; 0,732$ & $-21,409$ \\
\hline
\end{tabular}

enregistrée pour le cas A9, la plus petite valeur correspond au cas A4. En considérant la portée avec les cas A8 et A9 (l'axe de pignon s'incline avec variation de l'angle primitif), la ligne de contact ne se trouve plus au milieu de la portée (Figs. 9a et 10a). Elle se déplace en fonction du signe de variation de l'angle de rotation. Les ellipses de contact ne sont pas placées symétriquement par rapport à la ligne de contact. La méthode classique de Hertz ne sera pas appropriée au calcul de déformation, car elle considère que les ellipses de contact sont symétriques.

La correction en hauteur sans désalignement d'axes (cas B1) donne une erreur cinématique nulle à cause des points conjugués. La portée a une forme longitudinale qui reste inchangée avec les désalignements. Dans certains cas B3, B7 et B9, la ligne de contact dévie vers le haut de la denture menante au début du contact, 


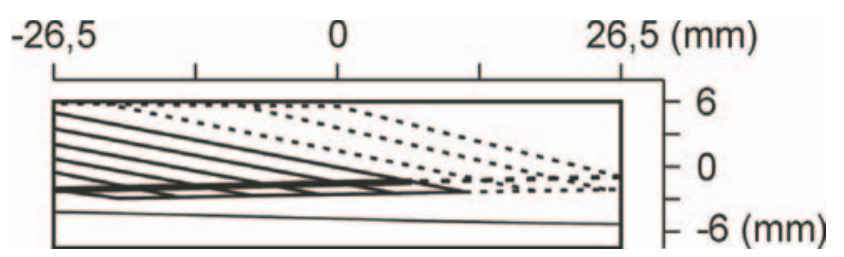

(a)

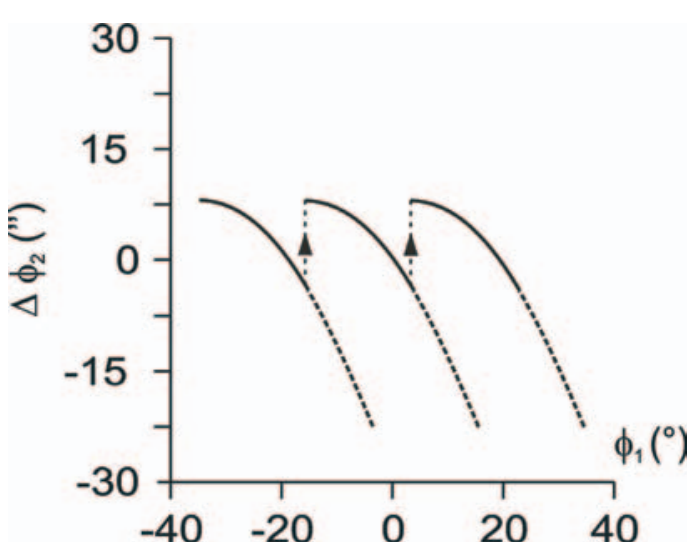

(b)

Fig. 11. Visualisation des résultats pour le cas B8. (a) Portée. (b) Erreur cinématique.

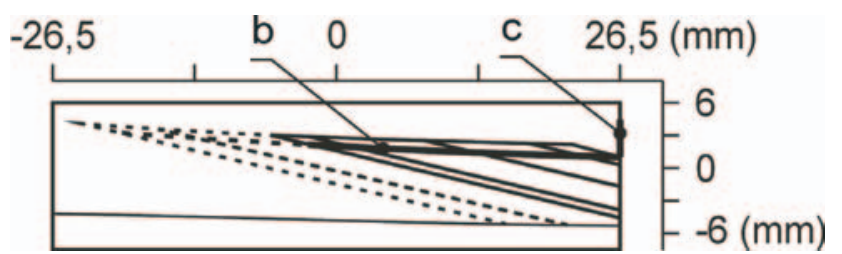

(a)

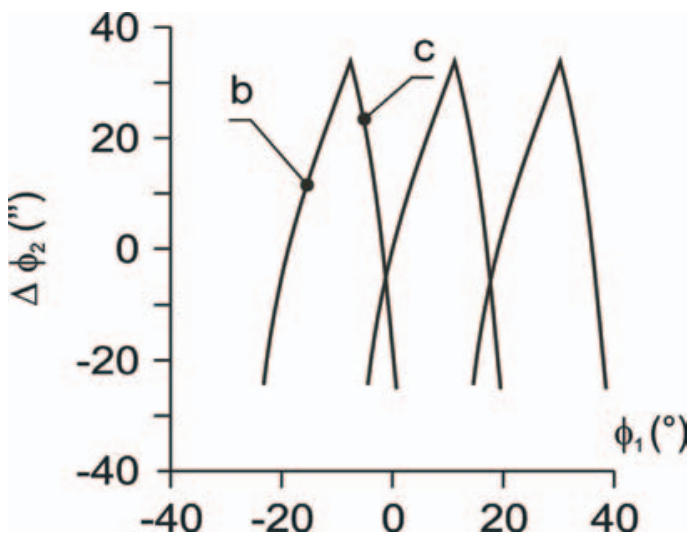

(b)

Fig. 12. Visualisation des résultats pour le cas B9. (a) Portée. (b) Erreur cinématique.

et le contact est obtenu sur les crêtes au gros bout de la surface de denture. Les figures correspondant aux cas B2B7 sont présentées dans l'Annexe B. Les désalignements à valeurs négatives déplacent la portée vers la tête des dents du pignon (cas B3, B5, B7 et B9 du Tab. 6) (voir Fig. 12a). Dans les cas B2, B4, B6 et B8 les déplacements ont lieu vers la partie basse (Fig. 11a). Les désalignements conduisent à une fonction discontinue de l'erreur cinématique. Le déplacement de la portée et la forme de l'erreur cinématique dépendent de la direction du déplacement.

La roue menée est en retard par rapport à la vitesse angulaire idéale si son déplacement axial est positif (cas B2, B4 e B6). Dans les cas B3, B7 et B8, la roue est en avance par rapport au pignon. Dans les cas de déplacement axial de la roue (cas B4 et B5), une forme parabolique de l'erreur cinématique est observée pour une très faible erreur cinématique. Dans le cas B5, le contact est continu et la valeur maximum de l'erreur cinématique $\Delta \phi_{2 \max }$ calculée est égale à $-0,912^{\prime \prime}$. L'intersection entre les courbes consécutives de l'erreur cinématique est très juste, mais si les surfaces se rapprochent, cette fonction est conservée. La rotation est négative (cas B9), le contact est continu, mais il est sur la crête, segment $c$ sur la figure $12 \mathrm{~b}$, et la valeur maximum de l'erreur cinématique $\Delta \phi_{2 \max }$ calculée est égale à $-38,846^{\prime \prime}$. La plus grande valeur du saut de l'erreur cinématique est enregistrée avec le cas B3 à 49,384". Le saut le plus faible correspond au cas B4 avec 3,240". Ces larges variations et le contact sur les crêtes aux extrémités de la surface de denture (cas B3, B7 et B9) montre la sensibilité aux désalignements. Par rapport au cas A $(225 \%)$, le taux de variation du saut de l'erreur cinématique est beaucoup plus élevé $(\times 21)$. En considérant la portée des cas B8 et B9 où l'axe de pignon s'incline, la ligne de contact ne se trouve plus au milieu de la portée (Figs. 11a et 12a). Ce phénomène est analogue à celui du cas A. La ligne de contact se déplace en fonction de l'angle de rotation. Les ellipses de contact ne sont pas symétriques.

\subsection{Déplacements axiaux et corrections suivant deux directions}

Afin de modifier la discontinuité de l'erreur cinématique, le flanc de denture est corrigé suivant deux directions. La forme de l'erreur cinématique est 


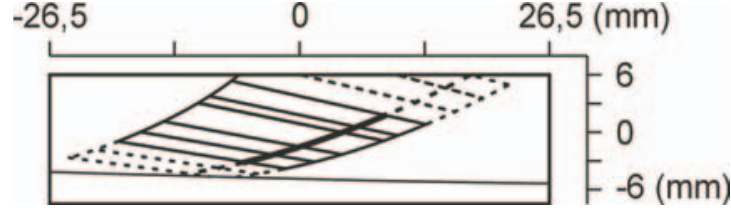

(a)

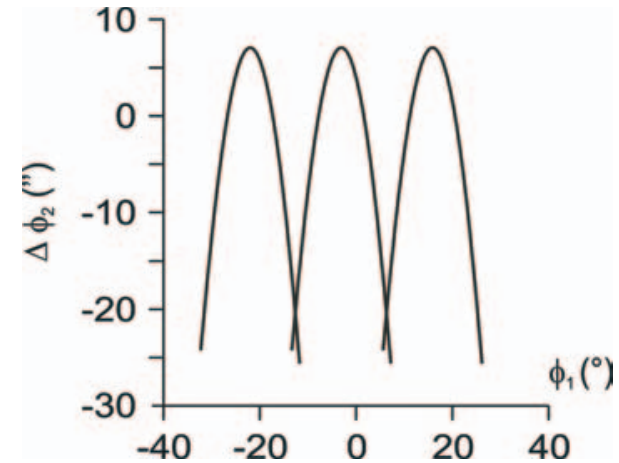

(b)

Fig. 13. Visualisation des résultats pour le cas C8. (a) Portée. (b) Erreur cinématique.

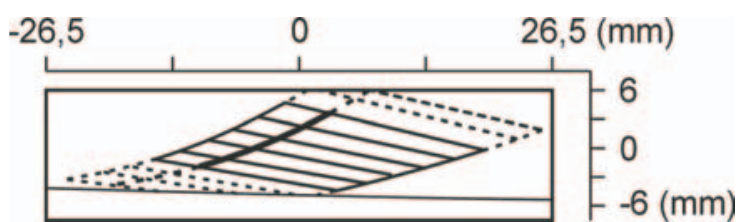

(a)

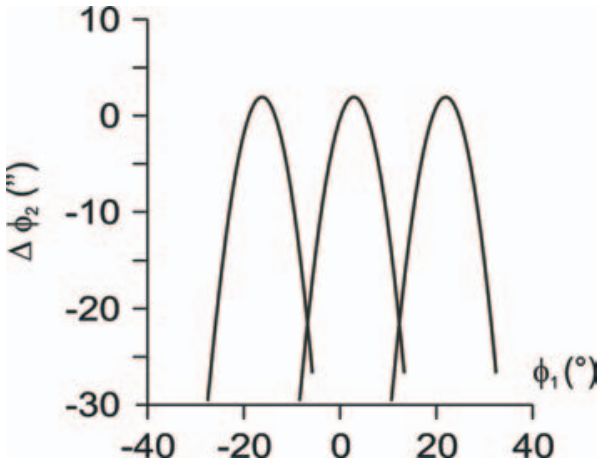

(b)

Fig. 14. Visualisation des résultats pour le cas C9. (a) Portée. (b) Erreur cinématique.

quasi-parabolique. À l'aide des réglages de la machine, une portée diagonale est obtenue (Fig. 7a). Ce résultat est inchangé par les désalignements des pièces (Figs. 13a et 14a). Les changements de position de la portée suivant la direction longitudinale sont déterminés originalement avec la correction en longueur pour le décalage hypoïde (résultats identiques des cas A2 et C2, aussi des cas A3 et C3 dans les premières colonnes des Tabs. 5 et 7). Les désalignements déplacent surtout la partie active de la portée suivant la direction de profil (Tab. 7) vers le segment haut (cas C5) et vers le segment de pied (cas C4) pour le pignon menant. Pour les autres cas, la portée est déplacée diagonalement, vers le petit bout et en haut dans les cas C3, C7 et C9, dans le sens inverse (vers le gros bout et en bas) dans les cas $\mathrm{C} 2$, C6 et $\mathrm{C} 8$. La forme quasi-parabolique de l'erreur cinématique est légèrement modifiée par les déplacements d'axes (Figs. 13b et 14b). De cette manière, la continuité de l'erreur cinématique est obtenue ainsi qu'une portée localisée. Les déplacements absolus de la partie active de la portée en hauteur sont inférieurs à ceux produits par la rotation de l'outil coupant. Le niveau maximal de l'erreur cinématique change peu en fonction du désalignement, $11,5 \%$ (Tab. 7). La ligne de contact n'est pas au milieu des ellipses de contact au cas C8 et C9 (Figs. 13a et 14a). On constate que ce phénomène est analogue dans tous les cas concernés par la variation de l'angle primitif.

\section{Conclusion}

Le contrôle des portées des engrenages spiro-coniques Klingelnberg est fait à partir de corrections des surfaces de denture directement obtenues sur la machine de taillage. L'influence des modifications des paramètres de taillage a été simulée. Les effets des désalignements des pièces en contact sur le comportement de l'engrènement, ont aussi été étudiés.

Le contact des surfaces corrigées dans une seule direction est très sensible à la variation de déplacement axial. La portée conserve sa forme originale sans déplacement axial, mais la fonction erreur cinématique n'est pas toujours continue. Le déplacement axial de la roue menée ajouté à la correction en hauteur peut induire une erreur cinématique continue, quasi-parabolique, mais le recouvrement entre les courbes consécutives est très limité. Si les surfaces se rapprochent, cette erreur cinématique est conservée mais l'amplitude de l'erreur cinématique augmente. Cette amplitude de l'erreur cinématique est très variable. La correction suivant la hauteur est plus sensible aux désalignements que la correction suivant la longueur. Ces problèmes d'engrènement sont réduits par les corrections suivant deux directions. La portée garde sa forme diagonale initiale en se localisant. La forme parabolique de l'erreur cinématique reste pratiquement constante. Les valeurs de déplacements axiaux ne sont pas suffisantes 


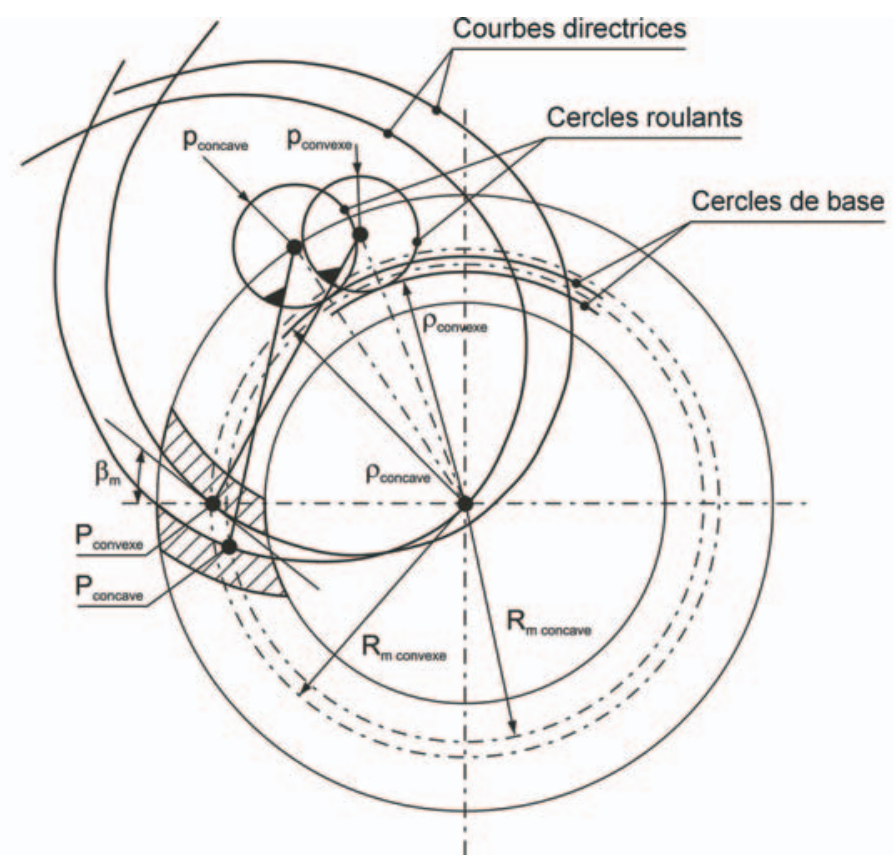

Fig. A.1. Système Cyclo-Palloïd Klingelnberg.

pour induire un saut d'erreur cinématique. La variation de l'angle primitif cause un changement de position des ellipses de contact autour la ligne de contact.
Remerciements. Nous tenons à remercier le Ministère de l'Éducation Nationale, de la Recherche et de la Technologie en France et le Ministère de l'Éducation en Hongrie (Numéro de donation : FKFP 0240/97) et également Ganz Dawid Brown Transmission Co. Budapest. Les remerciements vont aussi à l'Institut National des Sciences Appliquées de Lyon et l'Université des Sciences Techniques et Économiques de Budapest pour leur collaboration entre les Études Doctorales.

\section{Annexe A : Géométrie des dentures Klingelnberg}

Les dentures Klingelnberg sont générées par un outil coupant selon une procédure associée à la machine de taillage [12]. Le réglage de la machine sans correction produit des surfaces de denture conjuguées. La courbe directrice est une épicycloïde allongée générée par un mouvement de roulement sans glissement (Fig. A.1). La hauteur de denture est constante. Le point de référence $P$, pris au milieu de la denture, est situé à l'intersection de la courbure directrice et d'un cercle de rayon égal à la longueur de la génératrice moyenne. La surface de denture définitive est déterminée à la fois par la forme de la courbe directrice, la forme de l'outil coupant et son positionnement dans le support d'outil.

\section{Annexe B : Visualisation des résultats}

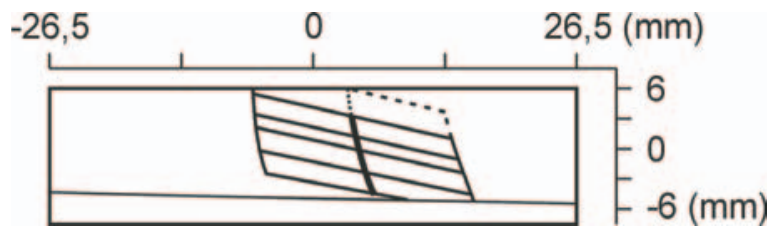

(a)

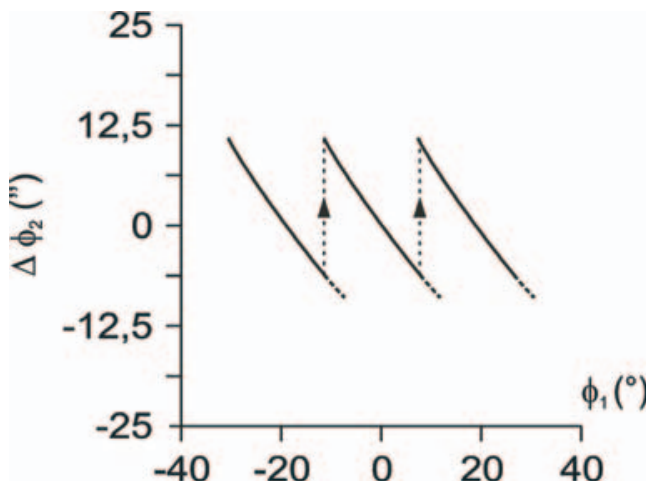

(b)

Fig. B.1. Visualisation des résultats pour le cas A2. (a) Portée. (b) Erreur cinématique. 


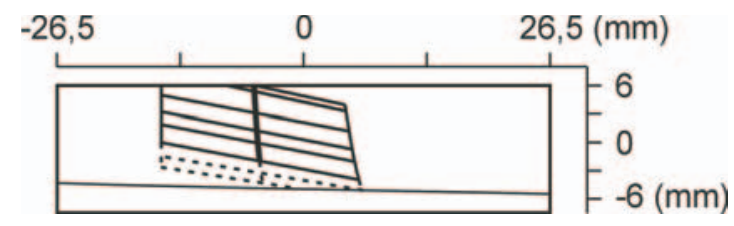

(a)

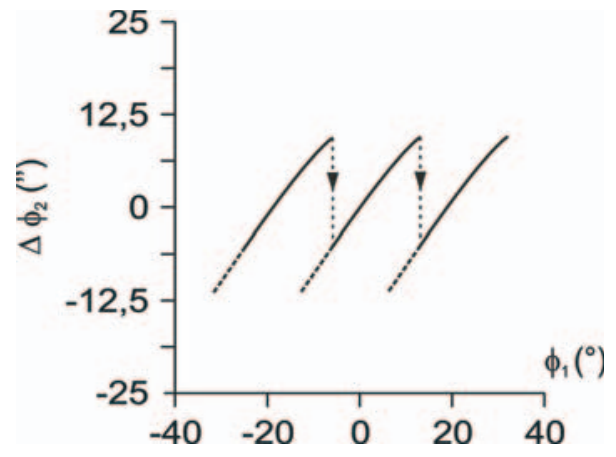

(b)

Fig. B.2. Visualisation des résultats pour le cas A3. (a) Portée. (b) Erreur cinématique.

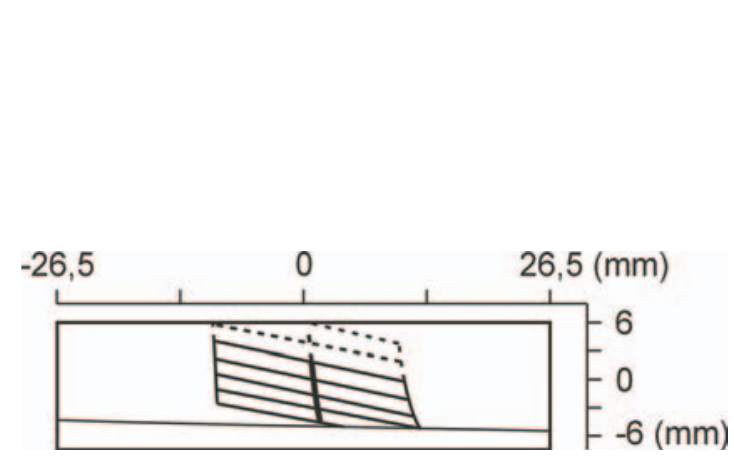

(a)

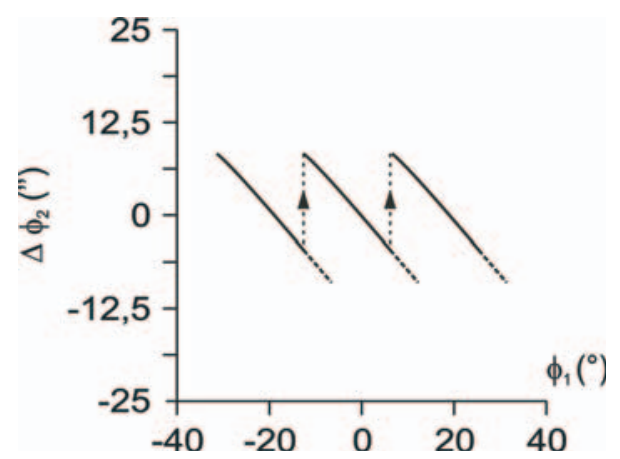

(b)

Fig. B.3. Visualisation des résultats pour le cas A4. (a) Portée. (b) Erreur cinématique.

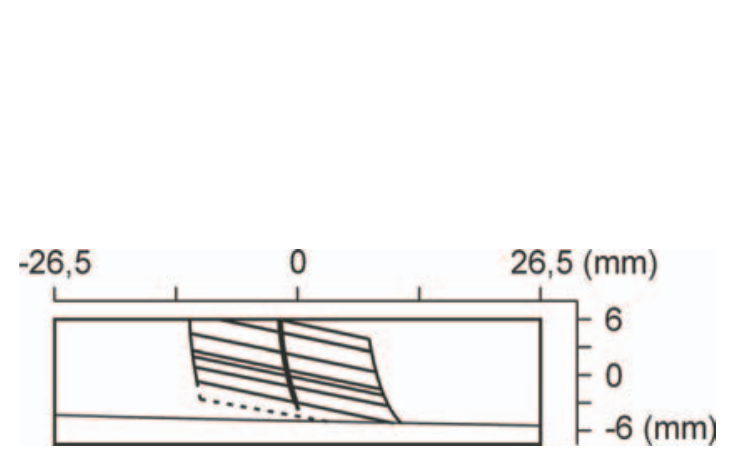

(a)

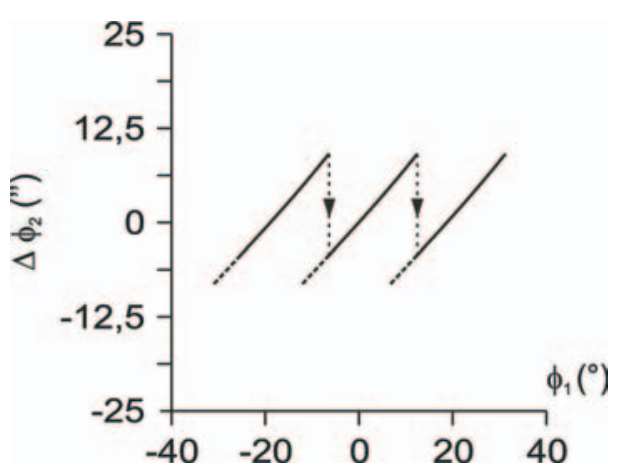

(b)

Fig. B.4. Visualisation des résultats pour le cas A5. (a) Portée. (b) Erreur cinématique.

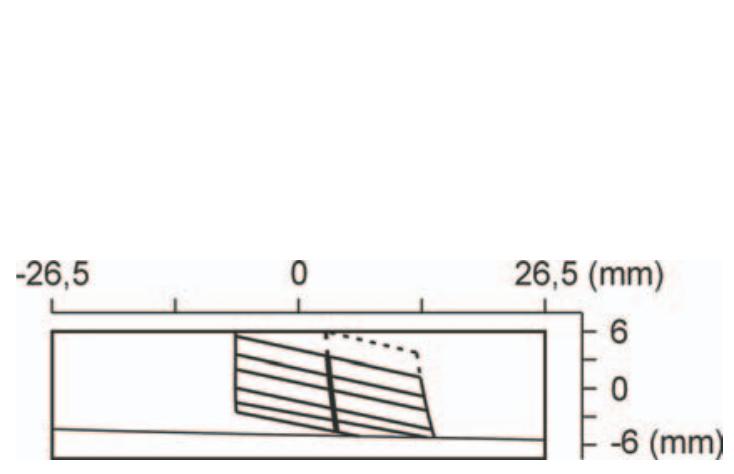

(a)

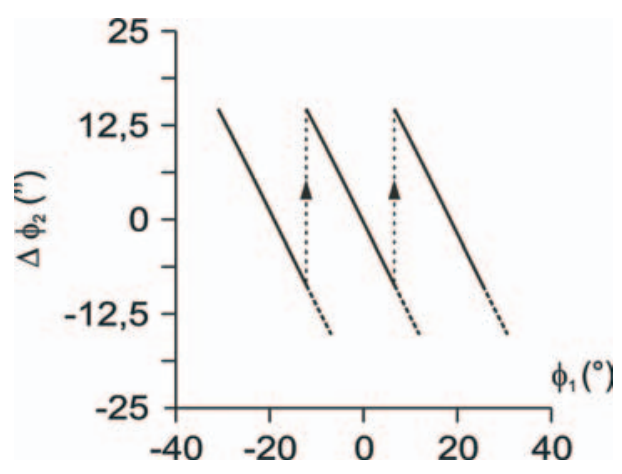

(b)

Fig. B.5. Visualisation des résultats pour le cas A6. (a) Portée. (b) Erreur cinématique. 


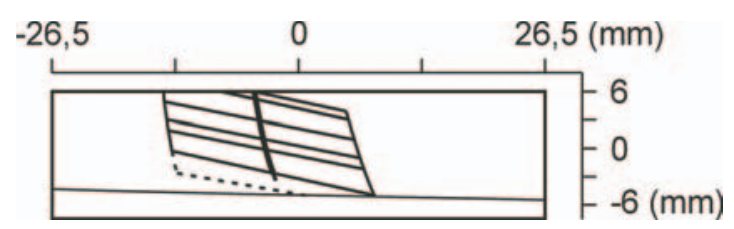

(a)

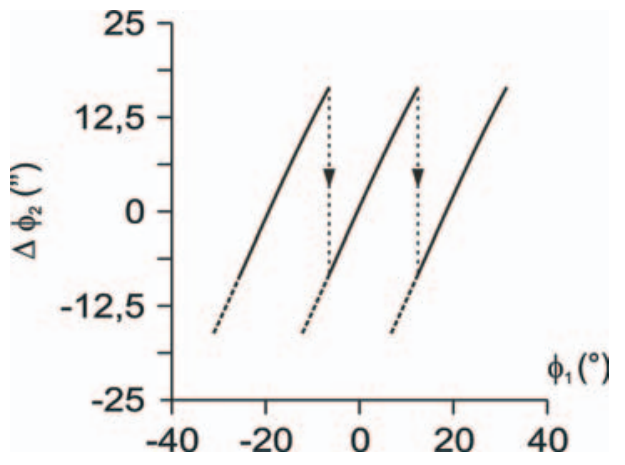

(b)

Fig. B.6. Visualisation des résultats pour le cas A7. (a) Portée. (b) Erreur cinématique.

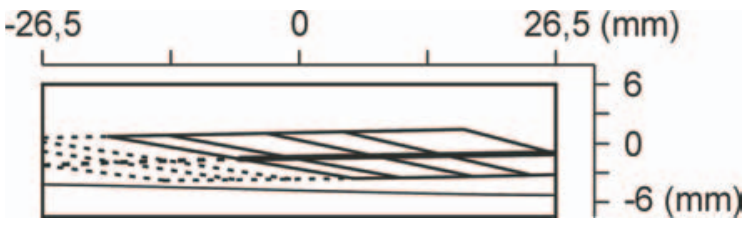

(a)

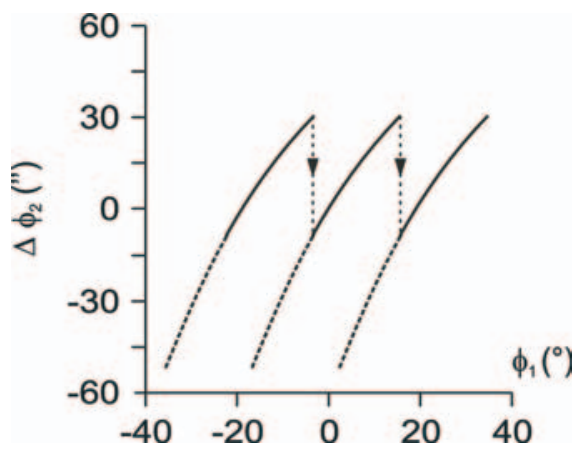

(b)

Fig. B.7. Visualisation des résultats pour le cas B2. (a) Portée. (b) Erreur cinématique.

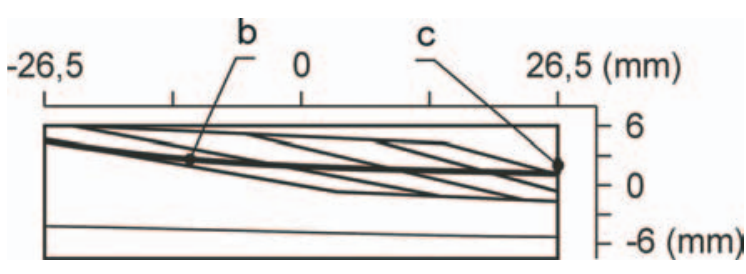

(a)

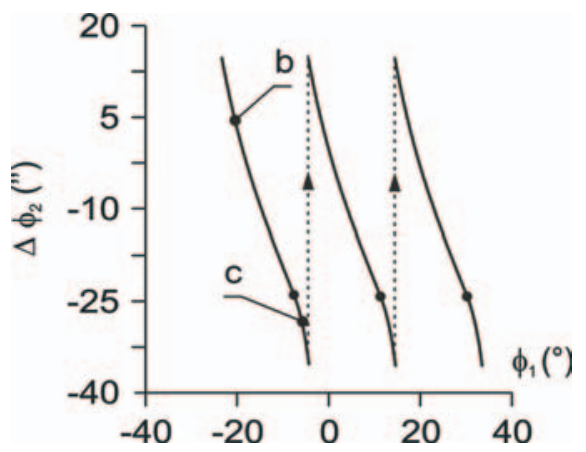

(b)

Fig. B.8. Visualisation des résultats pour le cas B3. (a) Portée. (b) Erreur cinématique.

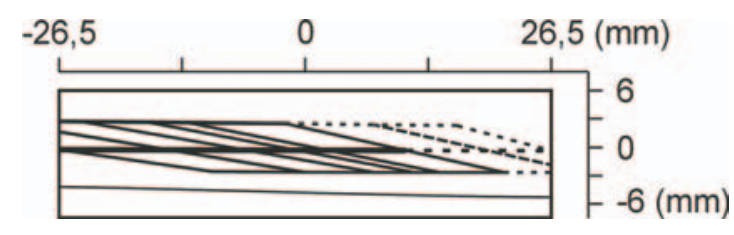

(a)

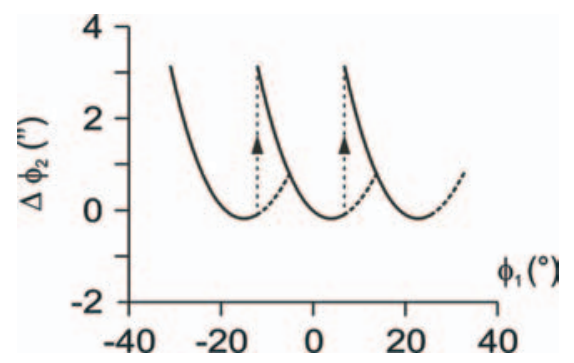

(b)

Fig. B.9. Visualisation des résultats pour le cas B4. (a) Portée. (b) Erreur cinématique. 


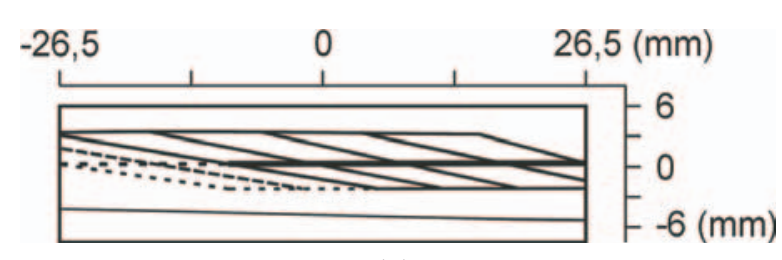

(a)

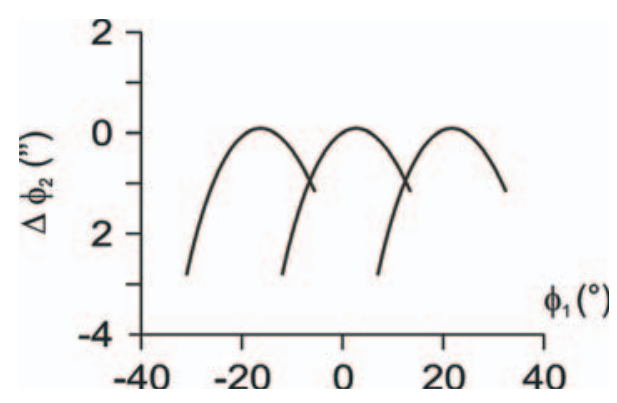

(b)

Fig. B.10. Visualisation des résultats pour le cas B5. (a) Portée. (b) Erreur cinématique.

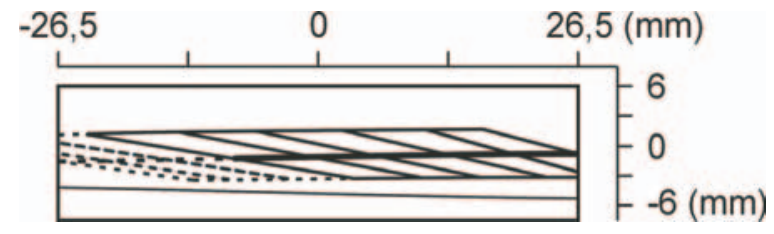

(a)

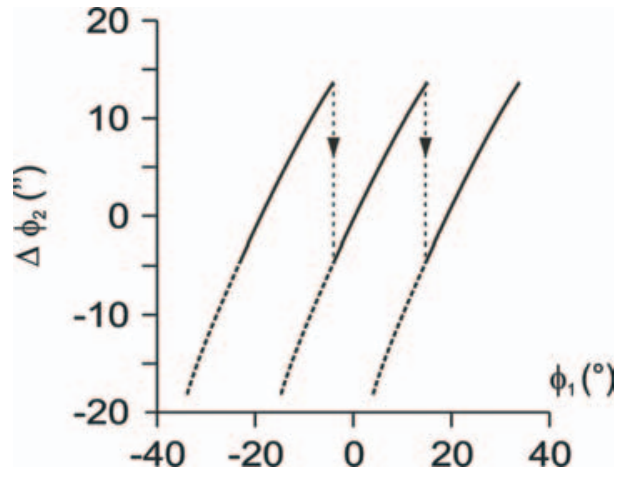

(b)

Fig. B.11. Visualisation des résultats pour le cas B6. (a) Portée. (b) Erreur cinématique.

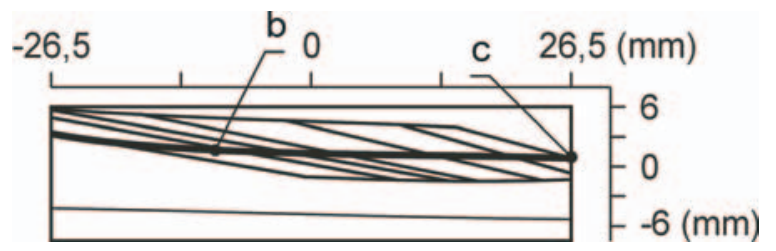

(a)

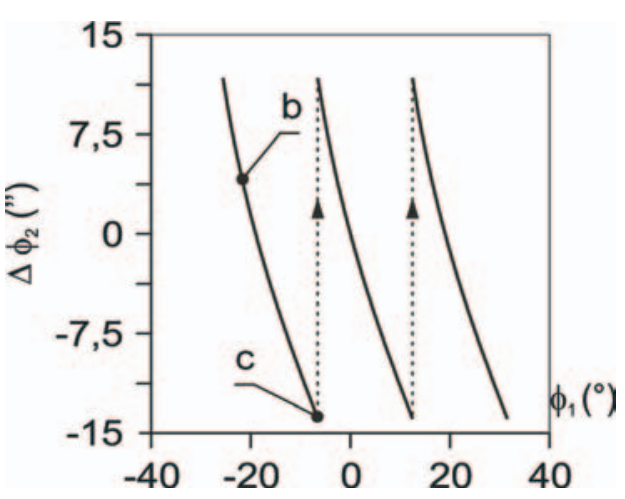

(b)

Fig. B.12. Visualisation des résultats pour le cas B7. (a) Portée. (b) Erreur cinématique.

\section{Références}

[1] C.J. Gosselin, L. Cloutier, The Generating Space for Parabolic Motion Error Spiral Bevel Gears Cut by the Gleason Method, ASME J. Mechanical Design 115 (1993) 483-489

[2] Z.H. Fong, Mathematical Model of Universal Hypoid Generator with Supplemental Kinematic Flank Correction Motions, ASME J. Mechanical Design 122 (2000) 136-142
[3] F.L. Litvin, J.C. Wang, R.F. Handschuh, Computerized Design and Analysis of Face-Milled, Uniform Tooth Height Spiral Bevel Gears Drives, ASME J. Mechanical Design 118 (1996) 573-579

[4] J. Marialigeti, J. Cseke, M. Lelkes, Connection of Some Cutting Parameters with Tooth Surface Modification in case of Epicycloidal Spiral Bevel Gears, Proceedings of Second Conference on Mechanical Engineering, Budapest 2 (2000) 587-591 
[5] Y. Zhang, F.L. Litvin, N. Maruyama, R. Takeda, M. Sugimoto, Computerized Analysis of Meshing and Contact of Real Tooth Surfaces, ASME J. Mechanical Design 116 (1994) 677-682

[6] M. Guingand, J.-P. DeVaujany, C. Cheval, D. Play, Influence of Design Parameters and Tooth Profile Modification for Reducing Gear Transmission Error, ASME IDECT/CIE 8th International Power Transmission and Gearing Conference, Baltimore, 2000, DETC 2000/PTG-14424

[7] K. Kavasaki, H. Tamura, Y. Nakano, A Method for Inspection of Spiral Bevel Gears in Klingelnberg CykloPalloid System, Proceedings of the 1994 International Gearing Conference, Newcastle upon Tyne, 1994, 305-310
[8] Z.H. Fong, C.B. Tsay, Kinematical Optimization of Spiral Bevel Gears, ASME J. Mechanical Design 114 (1992) 498-506

[9] Y. Zhang, F.L. Litvin, R.F. Handschuh, Computerized Design of Low-Noise Face-Milled Spiral Bevel Gears, Mechanism and Machine Theory 30 (1995) 1171-1178

[10] M. Lelkes, D. Play, J. Márialigeti, Cutting Parameters Definition for Klingelnberg Spiral Bevel Gears Optimization, Proceedings of The JSME International Conference on Motion and Power Transmissions MPT2001-Fukuoka 1 (2001) 375-380

[11] M. Lelkes, Définition des Engrenages Klingelnberg, Thèse de Doctorat Ingénieur : Institut National des Sciences Appliquées de Lyon, 02 ISAL 012, 2002

[12] F.L. Litvin, Theory of Gearing, NASA Reference Publication 1212, 1989 